\title{
Microlensing towards M 31 with MDM data
}

\author{
S. Calchi Novati ${ }^{1}$, Ph. Jetzer ${ }^{1}$, G. Scarpetta ${ }^{2}$, Y. Giraud-Héraud ${ }^{3}$, J. Kaplan ${ }^{3}$, \\ S. Paulin-Henriksson ${ }^{3}$, and A. Gould ${ }^{4}$
}

\author{
${ }^{1}$ Institute of Theoretical Physics, University of Zürich, Winterthurestrasse 190, 8057 Zürich, Switzerland \\ 2 Dipartimento di Fisica "E.R. Caianiello", Università di Salerno, via S. Allende, 84081 Baronissi (Sa), \\ and INFN Sez. di Napoli, Gruppo Collegato di Salerno, Italy \\ 3 Physique Corpusculaire et Cosmologie, Collège de France, 11 place M. Berthelot, 75231 Paris, France \\ ${ }^{4}$ Department of Astronomy, Ohio State University, Columbus, OH 43210, USA
}

Received 11 March 2003 / Accepted 18 April 2003

\begin{abstract}
We report the final analysis of a search for microlensing events in the direction of the Andromeda galaxy, which aimed to probe the MACHO composition of the M 31 halo using data collected during the 1998-1999 observational campaign at the MDM observatory. In a previous paper, we discussed the results from a first set of observations. Here, we deal with the complete data set, and we take advantage of some INT observations in the 1999-2000 seasons. This merging of data sets taken by different instruments turns out to be very useful, the study of the longer baseline available allowing us to test the uniqueness that is characteristic of microlensing events. As a result, all the candidate microlensing events previously reported turn out to be variable stars. We further discuss a selection based on different criteria, aimed at the detection of short-duration events. We find three candidates whose positions are consistent with self-lensing events, although the available data do not allow us to conclude unambiguously that they are due to microlensing.
\end{abstract}

Key words. methods: observational - methods: data analysis - cosmology: dark matter - cosmology: gravitational lensing cosmology: observations - galaxies: individual: M 31

\section{Introduction}

Gravitational microlensing is a powerful tool for the detection of dark matter in galactic haloes in the form of MACHOs (Paczyński 1986). Intensive searches in our Galaxy have shown that up to $20 \%$ of the halo could be formed by objects of around $M \sim 0.4 M_{\odot}$ (Alcock et al. 2000; Lasserre et al. 2000). This result is still debated (e.g. Jetzer et al. 2002), and remains to be confirmed. For this purpose it is useful to probe the MACHO distribution along different lines of sight.

A survey of M 31, nearby and similar to our own Galaxy, can supply several insights into this problem. Briefly, it tests a different line of sight in our Galaxy, it allows one to probe M 31's own halo globally and finally, the inclination of the M 31 disk should give an unmistakable signature of microlensing events (Crotts 1992; Baillon et al. 1993; Jetzer 1994).

Several collaborations have undertaken a search for microlensing events towards M 31 in the past years: AGAPE (Ansari et al. 1999); MEGA (Crotts et al. 2000); POINTAGAPE (Aurière et al. 2001); WeCapp (Riffeser et al. 2001). In this same framework we have already reported on the analysis of a partial set of the MDM data in Calchi Novati et al. (2002, hereafter Paper I). Furthermore, the POINT-AGAPE

Send offprint requests to: S. Calchi Novati, e-mail: novati@physik.unizh.ch collaboration has given evidence of 4 short high $\mathrm{S} / \mathrm{N}$ ratio microlensing candidates (Paulin-Henriksson et al. 2002, 2003).

In the present paper we conclude the analysis of the MDM data started in Paper I. We complement the MDM data with INT observations taken by the POINT-AGAPE collaboration. Furthermore, we discuss the issue of selection criteria for microlensing events, especially with respect to the background of variable stars, and we present results from an analysis based on different criteria. In Sect. 2 we summarize the observational setup and give some details of the data analysis, dealing in particular with some improvements in the selection procedure; in Sect. 3 we introduce the INT data and discuss how we use the longer baseline they give us to construct a strong supplementary selection criterion; Sect. 4 is devoted to the results of the analysis; in Sect. 5 we present our conclusions.

\section{Observations and data analysis}

\subsection{Observational setup}

We analyse data collected on the $1.3 \mathrm{~m}$ McGraw-Hill Telescope, at the MDM observatory, Kitt Peak (USA), towards the two sides of M 31 including the bulge ${ }^{1}$. Two fields are observed, located in $\alpha=00 \mathrm{~h} 43 \mathrm{~m} 24 \mathrm{~s}, \delta=41^{\circ} 12^{\prime} 10^{\prime \prime}(\mathrm{J} 2000)$

\footnotetext{
1 The data are shared with the MEGA collaboration.
} 
("Target", whose data have been the object of the analysis presented in Paper I) and $\alpha=00 \mathrm{~h} 42 \mathrm{~m} 14 \mathrm{~s}, \delta=41^{\circ} 24^{\prime} 20^{\prime \prime}(\mathrm{J} 2000)$ ("Control"). The Target field is centred on the far side of M 31 while the Control field is centred on the near side. Throughout the observations a $2048 \times 2048$ pixel CCD camera, with a field of view of $17^{\prime} \times 17^{\prime}$, has been used.

Two filters, similar to standard $R$ and $I$ Cousins, have been used in order to test achromaticity. This colour information gives us the chance of having a better check on red variable stars, which can contaminate the search for microlensing events.

We analyse data taken starting from October 1998 to the end of December 1999. While the baseline of the Control field is the same as that of the Target field, the Control field has only about 20 nights of observations for both filters ${ }^{2}$, which is only about half as much data as the Target field. The average value of the seeing is $\sim 1^{\prime \prime}$. 6 .

The photometric calibration is done with respect to a sample of reference secondaries identified in our frame (Magnier et al. 1992). As the photometric conditions for the reference images in the two fields are similar, the zero point calibrations reported in Paper I still hold.

\subsection{Reduction and analysis}

The reduction procedure and the candidate selection were discussed in detail in Paper I. Here we just recall the basic points and discuss in more detail some aspects that have since been improved.

\subsubsection{Pixel lensing}

Due to the distance of the target, the potential sources of microlensing events are not resolved stars. We use the pixel lensing technique developed by the AGAPE group to detect flux variations of unresolved sources (Ansari et al. 1997) ${ }^{3}$. In order to cope with photometric and seeing variations, we first choose for each filter a reference image (the $R$ reference image is also the geometric reference for both filters) and then calibrate the flux $^{4}$ of all other images with respect to the reference image by means of a linear correction. Our seeing correction is empirical and does not require the evaluation of the PSF of the image (Paper I).

\subsubsection{Bump detection}

Following Paper I, we look for bumps (in $R$ band) by a statistical analysis of the light curve. To this end we construct the

\footnotetext{
${ }^{2}$ Each night $\sim 20(11)$ images are taken in the $R(I)$ filter, which are then averaged to get the single image per night that we use in the following analysis.

3 An alternative method based on image subtraction is currently used by the MEGA collaboration (Tomaney \& Crotts 1996).

${ }^{4}$ With the notable exception of geometrical alignment, throughout the analysis we substitute for the pixel value the sum of the fluxes taken in a square of $5 \times 5$ pixels around the central pixel, i.e., the corresponding superpixel value.
}

two estimators for the significance of a variation $L$ and $Q$. We define

$L=-\ln \left(\Pi_{j \in \text { bump }} P\left(\Phi \mid \Phi>\Phi_{j}\right)\right)$ given $\bar{\Phi}_{\mathrm{bkg}}, \sigma_{j}$,

where

$P\left(\Phi \mid \Phi>\Phi_{j}\right)=\int_{\Phi_{j}}^{\infty} \mathrm{d} \Phi \frac{1}{\sigma_{j} \sqrt{2 \pi}} \exp \left[-\frac{\left(\Phi-\bar{\Phi}_{\mathrm{bkg}}\right)^{2}}{2 \sigma_{j}^{2}}\right] ;$

$\Phi_{j}$ and $\sigma_{j}$ are the flux and its associated error in a superpixel at time $t_{j}, \bar{\Phi}_{\text {bkg }}$ is an estimator of the baseline level, and a "bump" is defined as a variation with at least 3 consecutive points more than $3 \sigma$ above the baseline. We define

$Q \equiv \frac{\chi_{\text {const }}^{2}-\chi_{\text {pacz }}^{2}}{\chi_{\text {pacz }}^{2} / \text { d.o.f. }}$

where $\chi_{\text {const }}^{2}$ is the $\chi^{2}$ calculated with respect to the constantflux hypothesis and $\chi_{\text {pacz }}^{2}$ is the $\chi^{2}$ calculated with respect to a Paczyński fit. We stress that $Q$ is evaluated along the entire light curve, while $L$ is evaluated only along the bump.

In Paper I we carried out the analysis to detect clusters of variable light curves using $L$. We eventually retained from the sample of selected light curves (the ones with the highest value of $L$ in a given cluster) only those characterized by $Q>Q_{\text {thresh }}$.

Here we follow a somewhat different approach in that we select clusters related to flux variations by demanding $Q>$ $Q_{\text {thresh. }}$. Compared to the selection based on $L$, we are biased in favor of monobump-shape variations. Moreover, we exclude all those light curves showing spurious variations for which a high value of $L$ can happen to be induced, e.g., by an underestimation of the baseline level.

To conclude with the bump detection, we evaluate for all the pixels in each cluster $L$, and we then proceed as in Paper I. This is a better approach because $L$ is based on an evaluation of the baseline more appropriate to this purpose, that is, to selecting the light curve showing the maximum flux deviation from the baseline.

\subsubsection{Shape analysis}

For microlensing events the flux variation must be unique, it must follow (in the point-like and uniform-motion approximations) the symmetric Paczyński shape, and it must be achromatic.

The flux as a function of time for an event with amplification $A(t)$ and an unlensed source $\phi^{*}$ can be written as

$\phi(t)=\phi_{\mathrm{bkg}}+[A(t)-1] \phi^{*}$,

where $\phi_{\text {bkg }}$ is the background flux including $\phi^{*}$.

The achromatic shape analysis is carried out as in Paper I. We perform a 7-parameter Paczyński fit to the two bands simultaneously (background level $\phi_{\mathrm{bkg}}$, source flux without amplification $\phi^{*}$, and the parameters of the amplification: time of maximum amplification $t_{0}$, Einstein time $t_{\mathrm{E}}$, and the impact parameter $u_{\min }$ ). In the absence of direct knowledge of the source flux from e.g., a Hubble Space Telescope (HST) image (e.g., Aurière et al. 2001) or an indirect measurement of it from a high signal-to-noise ratio lightcurve (e.g. 
Paulin-Henriksson et al. 2003), there is a degeneracy among the three parameters $\phi^{*}, t_{\mathrm{E}}$, and $u_{\min }$ (Gould 1996). For these cases we retain two "effective parameters", the time width $t_{1 / 2}=t_{1 / 2}\left(t_{\mathrm{E}}, u_{\min }\right)$ and the excess at maximum with respect to the background $\Delta \phi_{\max }=\Delta \phi_{\max }\left(\phi^{*}, u_{\min }\right.$ ) (from which we evaluate the "magnitude at maximum" $R_{\max }$ and the colour at maximum $\left.(R-I)_{\mathrm{C}}\right)$. For an event to be achromatic we have to test whether, along the bump, the ratio of the deviation of the flux from the background in the two bands remains constant in time $\left(\Delta \phi_{R} / \Delta \phi_{I}=\phi_{R}^{*} / \phi_{I}^{*}=\text { constant }\right)^{5}$.

Furthermore, we carry out the Durbin-Watson test (Durbin \& Watson 1951), which is sensitive to time correlation among consecutive residuals. In effect, it is able to check whether consecutive points lie, for instance, all above or below the best Paczyński fit shape, such behaviour being characteristic of variable stars (and for which the $\chi^{2}$ test can say little). The test is based upon the evaluation of a Durbin-Watson coefficient, $\mathrm{d} w$, which must lie in an interval that depends on the number of points along the light curve, e.g. for 20 points it must be $1.41(1.15)<\mathrm{d} w<2.59(2.85)$ at the $10 \%$ (2\%) level of significance.

\section{The baseline of INT data as a test for bump uniqueness}

\subsection{The problem of variable sources}

A main problem in the search for microlensing events is the estimate of the background noise given by variable sources. This is particularly serious in the case of pixel lensing for two main reasons. First, the class of stars to which we are in principle most sensitive are the red giants, for which a large fraction are variable stars (regular or irregular). Second, as we look for pixel flux variations, it is always possible to collect (in the same pixel) light from more than one source whose flux is varying.

Thus, in the analysis we are faced with two problems: large-amplitude variable sources whose signal can mimic a microlensing signal, and variable sources of smaller amplitude whose signal can give rise to non-Gaussian fluctuations superimposed on the background or on other physical variations.

In Paper I we followed a sufficiently conservative approach to minimize the impact of these problems. We adopted severe criteria in the shape analysis with respect to the Paczyński fit (stringent cut for both the $\chi^{2}$ and the Durbin-Watson test) and, furthermore, we eliminated candidates with both a long timescale $\left(t_{1 / 2}>40\right.$ days $)$ and a red colour $\left((R-I)_{\mathrm{C}}>1\right)$, since these most likely originate from variable stars.

As we had already noted, this analysis suffered from the intrinsic limitation of an insufficient baseline, so that the variablesource issue could not be conclusively tested.

\footnotetext{
5 Note that this same test can be applied to resolved-source microlensing, provided that "background" is replaced by "baseline", i.e. the combined flux from the true source plus any unresolved blended companions. That is, blending breaks achromaticity only for the ratio of the total fluxes in two bands, not differences in these fluxes from baseline.
}

Here we take advantage of the opportunity afforded by access to reduced INT data to extend our selected light curves. As we show, this will be a key element of our analysis.

\subsection{INT extension of MDM light curves}

The INT fields almost completely cover the MDM fields (except for a sizable fraction of the Control field far from the M 31 bulge and a narrow band in the Target field). The good sampling during the two INT seasons 1999-2000 of data acquired in Sloan $r^{\prime}$ and $i^{\prime}$ bands ${ }^{6}$, together with their high quality, allows a straightforward and quantitative analysis.

As a first step we have calculated the astrometric ${ }^{7}$ transformations between the different fields (data from 4 different INT CCDs are needed to cover the two MDM fields). We found that a global transformation over one entire field led to systematic errors as large as $5^{\prime \prime}$. Hence, we use our large samples of 1194 and 845 references stars in the Target and Control field, respectively, to make a linear (i.e., 6-parameter) local transformation for each selected pixel. We demand a minimum of 24 reference stars, which are usually found within a square of $\sim 2^{\prime}-3^{\prime}$ about the selected pixel. We thereby reach an average astrometric precision of $\sim 0$ !' 2 . To this we add the error in locating the center of the variation, so that on average we get $\sigma \sim 0.5$.

As a second step we have determined from the crossanalysis of about 30 selected resolved stars in each field, a "colour equation" that allows us to align the MDM ( $R$ and $I$ ) and INT $\left(r^{\prime}\right.$ and $\left.i^{\prime}\right)$ instrumental magnitudes closely enough to permit a quantitative comparison of the relative flux variations in each light curve.

The uniqueness condition for a given variation requires that the extension of the light curve into the INT data should remain flat. However, the analysis of the PA-N1 event (Aurière et al. 2001) has shown that a microlensing light curve may be contaminated by a neighbouring variable star. Therefore, care must be taken before rejecting a microlensing event because of a second bump, and it is essential to test the following issues: the relative astrometric position of the two bumps and the similarity of the shape of the two deviations.

Given a candidate microlensing light curve in the MDM data, we locate the corresponding INT light curve, and then calculate the estimator $L$ in a square of $7 \times 7$ pixels around the central pixel to check whether there is a variation, and where it is located. If we detect a variation in the INT data, we calculate its amplitude and width with a Paczyński fit in order to compare quantitatively the two variations.

As a criterion of rejection for a given MDM candidate microlensing event for which we find a variation on its extension into the INT data, i.e., a likely variable star, we demand that the position of the INT variation be compatible with the corresponding MDM variation within $3 \sigma$ and that the relative widths

\footnotetext{
6 For this analysis we do not use the available $g^{\prime}$ band data.

7 The INT pixel size is 0.33 versus 0.50 for MDM pixels.
} 

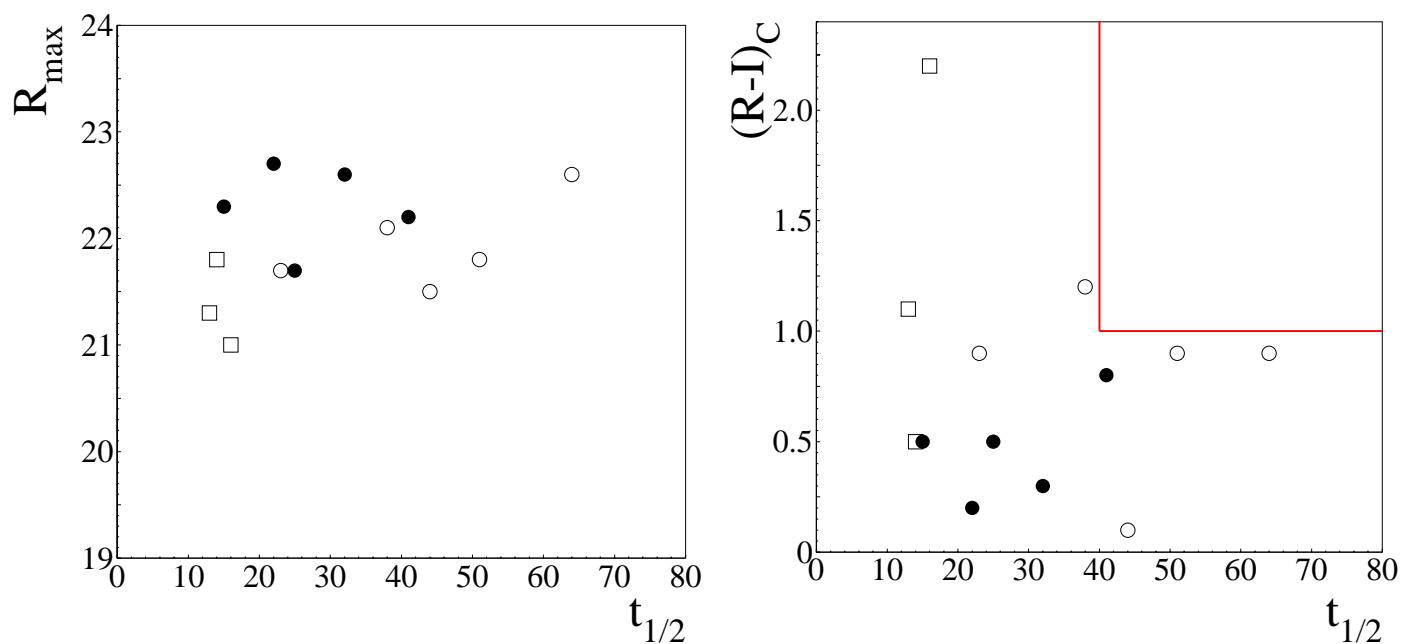

Fig. 1. Physical characteristics for the selected light curves in Sect. 4.1 (circles). Filled symbols represent candidates previously reported in Target field. Squares represent the result of the analysis presented in Sect. 4.2. In the right panel, the lines at $t_{1 / 2}=40$ days and $(R-I)_{\mathrm{C}}=1$ indicate our excluded region of this parameter space.

Table 1. Results of the stability analysis on the INT data extension of the selected MDM microlensing candidates. For each MDM light curve ( $\mathrm{T}$ and $\mathrm{C}$ stand respectively for Target, where we use the same numeration used in Paper I, and Control) for which we find an astrometrically compatible INT bump, we report the number of standard deviation within which the position and the bump parameters (duration and flux deviation in both bands as calculated from independent Paczyński fits on each of the MDM and INT light curves), of the two variations are compatible.

\begin{tabular}{ccccc}
\hline \hline id & $\sigma($ position $)$ & $\sigma\left(t_{1 / 2}\right)$ & $\sigma\left(\Delta \Phi_{R}\right)$ & $\sigma\left(\Delta \Phi_{I}\right)$ \\
\hline $\mathrm{T} 1$ & 1 & 1 & 2 & 2 \\
$\mathrm{~T} 2$ & 1 & 1 & 1 & 2 \\
$\mathrm{~T} 3$ & 1 & 4 & 4 & 3 \\
$\mathrm{~T} 4$ & 1 & 4 & 5 & 3 \\
$\mathrm{~T} 5$ & 1 & 3 & 2 & 3 \\
$\mathrm{~T} 6$ & 1 & 2 & 1 & 1 \\
\hline $\mathrm{C} 1$ & 1 & 1 & 2 & 4 \\
$\mathrm{C} 2$ & 1 & 2 & 2 & 2 \\
\hline
\end{tabular}

and deviations of flux lie within $6 \sigma$ as evaluated from the two independent Paczyński fits ${ }^{8}$.

\section{Candidate selection}

\subsection{Target and Control fields: The first selection}

We now present the results in both MDM fields, Target and Control, of an analysis complemented where possible by the stability test using the INT data.

${ }^{8}$ We allow here a larger margin because we are aware that probing such an effect can be difficult, especially when dealing with variations showing both a long time width and a red colour, these being possibly due to red variable stars that do not necessarily show a strictly periodic and regular behaviour.
The threshold value for the estimators of the significance of the bump are fixed as in Paper I $\left(Q>100\right.$ and $L_{1}>100$, but now we proceed as explained in Sect. 2.2.2 ${ }^{9}$ ). The selection criteria are the same as in Paper I: the selected light curves must have enough points along the bump (at least 4 points on both sides of the maximum, and 3 inside the interval $t_{0} \pm t_{1 / 2} / 2$ ); for the Paczyński fit we require that $\chi^{2} /$ d.o.f. $<1.5$ and that the Durbin-Watson test be satisfied at the $10 \%$ level; we require that either $t_{1 / 2}<40$ days or $(R-I)_{\mathrm{C}}<1$.

Due to the different approach we follow in bump detection, in the Target field we find 1 more light curve in addition to the 5 we already reported in Paper I. In the Control field we find 4 light curves compatible with microlensing.

We summarize the main physical characteristics of these 10 events in Fig. 1 , which shows $R_{\max }$ vs. $t_{1 / 2}$ and $(R-I)_{\mathrm{C}}$ vs. $t_{1 / 2}$.

As can be noted, with respect to the selected light curves in Paper I, we tend to lack short timescale candidates among the new events and, moreover, 3 of the 5 candidates lie at the boundary we have fixed for the colour-timescale compatibility with a microlensing signal.

The INT data allow us to check the stability of all 5 Target light curves and 2 of the 4 Control light curves. In Table 1 we summarize the results of this comparative analysis, relative astrometry and relative shape analysis.

As can be seen all 8 MDM events show compatible variations on their INT extensions. (In particular, we note that the positions of the two bumps are always compatible at $1 \sigma$ level). As a result, all the checked light curves are rejected as possible microlensing candidates. As an example, we show MDM light curves T4 and T5 (respectively, the shortest and the brightest flux variations detected) together with their INT extensions (Fig. 2).

This analysis shows how the sample of microlensing candidates derived from this selection is strongly contaminated by variable stars. The two additional Control light curves for

\footnotetext{
9 We note that, in principle, for a cluster of pixels with $Q>100$, we can have $L_{1}<100$.
} 

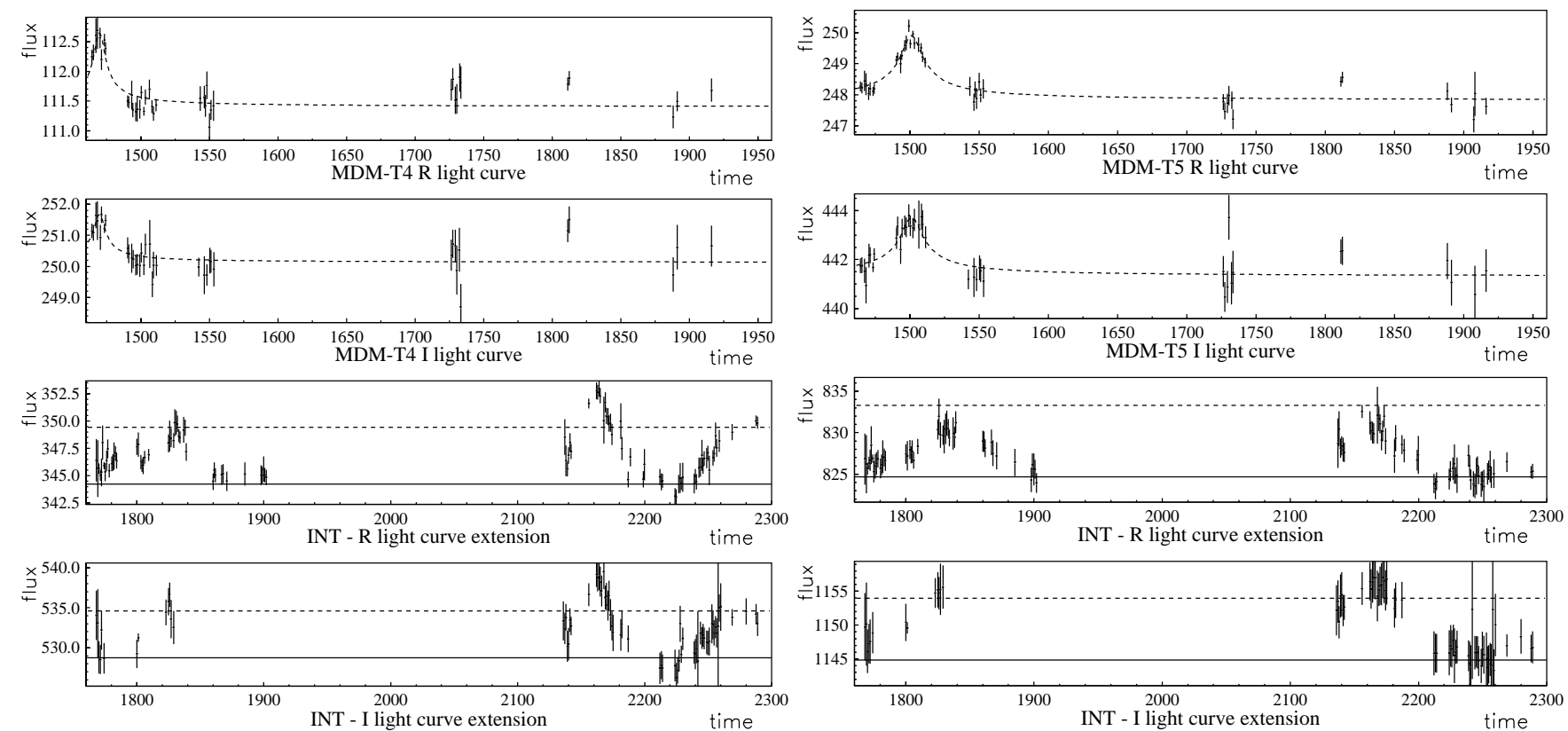

Fig. 2. MDM T4 (left) and T5 light curves together with their extensions into the INT data. On the $y$ axis, flux is in ADU/s; on the $x$ axis, time is in days, with the origin in J-2449624.5 (both data sets). For the MDM light curves the dashed line represent the result of the Paczyński fit. For the INT light curves, shown together with the solid line representing the baseline is a dashed line representing the level of the maximum deviation of flux found on the corresponding MDM light curve.

which there are no INT extensions have parameters: $t_{1 / 2}=$ $51 \pm 5,64 \pm 6$ days and $(R-I)_{\mathrm{C}}=0.9 \pm 0.2$, i.e., they are relatively long candidates. It then follows that, without INT vetting, it is no longer reasonable to look at them as viable microlensing candidates. Since, also looking at the results of the previous analysis, we suspect them to be red variable stars but cannot prove this without an extended baseline, we must exclude, when searching for such long variations, the non-INT portions of the MDM fields from the analysis.

\subsection{Search for short duration events}

The analysis carried out in the previous section shows that variable sources can mimic the Paczyński shape quite well, even with data available in two bands. It seems therefore appropriate in the search for viable microlensing candidates to relax the criteria introduced to characterize the shape of the variation and, on the other hand, to restrict the allowed space of physical parameters. We remark that the longer baseline now available make this approach viable. This search is also motivated by the lack of self-lensing events, which we would expect to find.

We then proceed to a new selection with the same threshold values for $L$ and $Q$. For the temporal sampling, we require at least 3 points inside the interval $t_{0} \pm t_{1 / 2} / 2$, with at least one on each side of $t_{0}$. We then impose the following conditions:

$-\chi^{2} /$ d.o.f. $<5$;

- DW test at $2 \%$ significance level;

$-t_{1 / 2}<20$ days ${ }^{10}$.

${ }_{10}$ We note that these short timescales are consistent with what we expect from the Monte Carlo simulations discussed in Paper I.
This search yields 8 additional light curves (4 in each field) which have durations $t_{1 / 2} \in(13,20)$ days and flux deviations $R_{\max } \in(21.0,22.8)$. The stability test on the INT data, as outlined in Sect. 3.2, allows us to reject 5 of these variations as microlensing candidates. We are left with three light curves, all lying in the Control field. We label these C3, C4 and C5, the last having no INT extension. The issue of the stability on the INT extension of the two remaining candidates deserves some additional comments.

MDM-C3: An INT variation is detected only in the $i^{\prime}$ light curve. Its position is compatible within $1 \sigma$ to that of the MDM variation. We note that the observed $i^{\prime}$ variation is significantly smaller than that observed on the MDM $I$ light curve and, as mentioned, the $r^{\prime}$ light curve is flat. Moreover, the time width of the MDM variation is actually quite short. This analysis indicates that, although sitting in the same position, the two variations may be due to different sources. At the same time we are aware that the INT data do not allow us to fully characterize the shape of the bump. We thus consider the stability test for this candidate to be inconclusive.

MDM-C4: We detect a variation on the INT data that we localize within $1 \sigma$ from the corresponding MDM variation. We evaluate the variations of flux in $r^{\prime}$ and $i^{\prime}$ as being compatible within 3 and $1 \sigma$ respectively with the corresponding variations on the MDM light curve. However, the observed time width of the INT variation, $t_{1 / 2} \sim 70$ days, is significantly larger than the evaluated time width of the MDM variation, $t_{1 / 2} \sim$ 13 days. As is the case for $\mathrm{C} 3$, the shape analysis may indicate that we are observing on the same light curve a variable star (a likely long period red variable) and a microlensing event. Our data are, however, insufficient to confirm or to reject this 
Table 2. Main characteristics of the three selected short-event light curves; $d$ is the projected distance from the centre of M 31 .

\begin{tabular}{cccc}
\hline \hline & $\mathrm{C} 3$ & $\mathrm{C} 4$ & $\mathrm{C} 5$ \\
\hline$\alpha(\mathrm{J} 2000)$ & $00^{\mathrm{h}} 42^{\mathrm{m}} 12.5^{\mathrm{s}}$ & $00^{\mathrm{h}} 42^{\mathrm{m}} 25.6^{\mathrm{s}}$ & $00^{\mathrm{h}} 41^{\mathrm{m}} 52.8^{\mathrm{s}}$ \\
$\delta(\mathrm{J} 2000)$ & $41^{\circ} 21^{\prime} 30^{\prime \prime}$ & $41^{\circ} 26^{\prime} 27^{\prime \prime}$ & $41^{\circ} 17^{\prime} 18^{\prime \prime}$ \\
$d$ & $8^{\prime} 01^{\prime \prime}$ & $10^{\prime} 53^{\prime \prime}$ & $9^{\prime} 45^{\prime \prime}$ \\
$\chi^{2} /$ d.o.f. & 2.90 & 2.32 & 2.47 \\
$\mathrm{~d} w_{R}$ & 2.48 & 2.01 & 1.78 \\
$\mathrm{~d} w_{I}$ & 1.62 & 2.23 & 1.92 \\
$t_{1 / 2}($ days $)$ & $16 \pm 2$ & $13 \pm 2$ & $14 \pm 2$ \\
$R_{\max }$ & $21.0 \pm 0.1$ & $21.3 \pm 0.1$ & $21.8 \pm 0.1$ \\
$R-I$ & $2.2 \pm 0.2$ & $1.1 \pm 0.2$ & $0.5 \pm 0.2$ \\
INT data & inconclusive & inconclusive & no extension \\
\hline
\end{tabular}
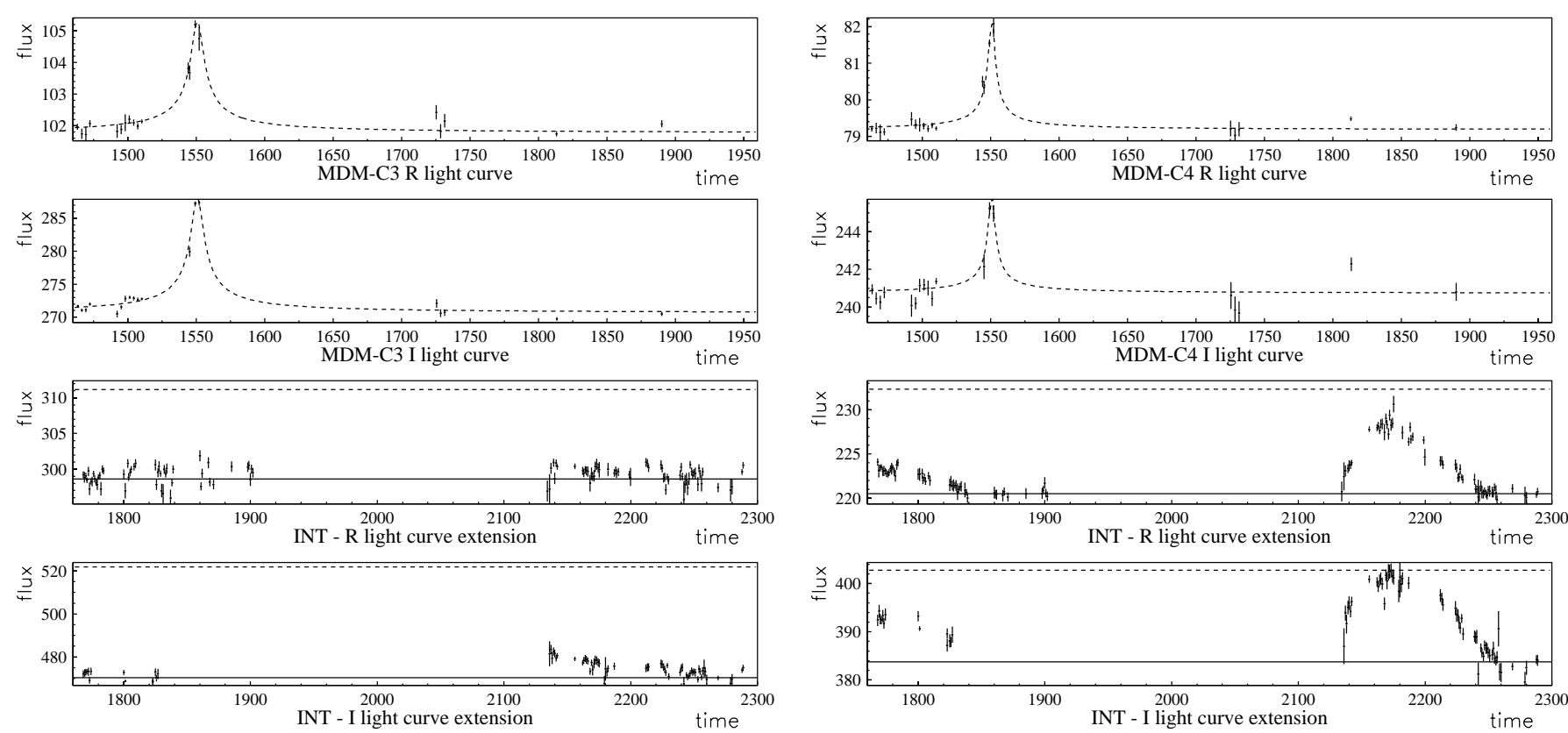

Fig. 3. The MDM-C3 light curve (top) and its corresponding INT extension. Notation is the same as in Fig. 2.

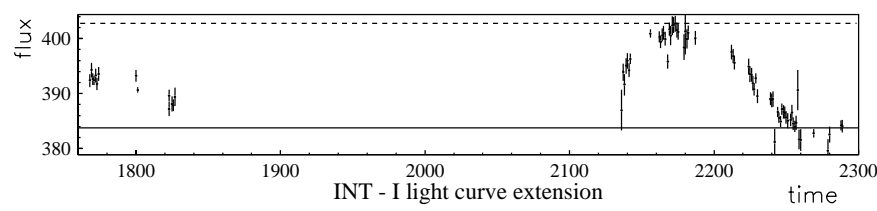

Fig. 4. The MDM-C4 light curve (top) and its corresponding INT extension. Notation is the same as in Fig. 2.

hypothesis. In this case too we then consider the stability test for this candidate to be inconclusive.

In Table 2 we report the main characteristics of these light curves, position and physical parameters as evaluated from the Paczyński fit. We note that, lacking any information from other sources (e.g. HST images) to measure the flux of the unamplified source, and since, on the basis of the fit alone, the data do not allow us to break the parameter degeneracy, we have no way to get any reliable information on the physical parameter $t_{\mathrm{E}}$, so that also no reliable estimate of the mass of the lens is possible.

As can be noted by looking at the light curves (Figs. 3-5), the sampling does not allow us to test conclusively the symmetry of the bump. Regarding the achromaticity, the ratio of $R$ and $I$ deviations from the baseline for the points belonging to the bump is about constant, as expected for microlensing events. We note that the MDM-C3 light curve, whose variation is quite

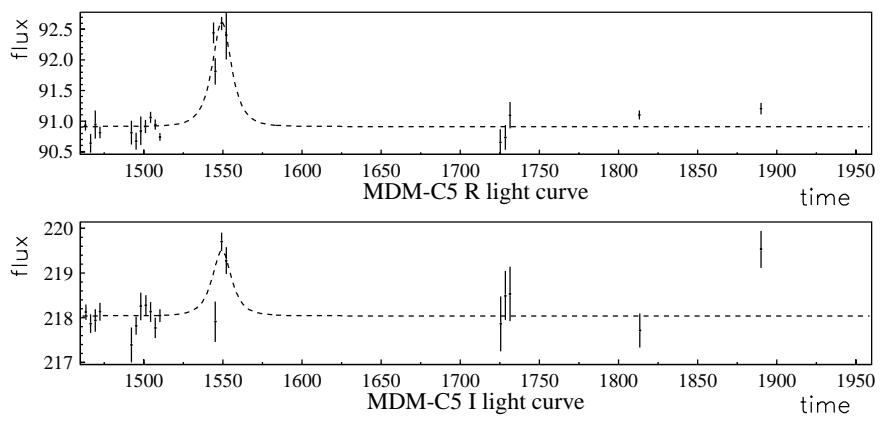

Fig. 5. The MDM-C5 light curve, for which no extension on INT data is available. Notation is the same as in Fig. 2.

short and very red, occupies a peculiar position in the $(R-I)_{\mathrm{C}^{-}}$ $t_{1 / 2}$ parameter space (Fig. 1, upper left of right panel). 
Even if the available data do not allow us to draw firm conclusions on the nature of the selected flux variations, their projected distance from the centre of M 31 (see Table 2) make them marginally consistent with the self-lensing events that we expect according to the Monte Carlo simulations discussed in Paper I.

\subsection{Comparison with POINT-AGAPE results}

In the framework of microlensing searches towards $\mathrm{M} 31$, the most promising results have been so far reported by the POINT-AGAPE collaboration. In particular, they have identified 4 high $\mathrm{S} / \mathrm{N}$ (likely to be microlensing) light curves with high flux variation, $R_{\max }<21$, and short timescale $\left(t_{1 / 2}<\right.$ 25 days, with 3 that are as small as $t_{1 / 2} \sim 2$ days), two of which lie quite near the M 31 bulge, where, however, data still do not allow one to answer unambiguously the question of whether they are due to self-lensing effects or to MACHOs (Paulin-Henriksson et al. 2003). We note that we lack, in the MDM data, such short duration and highly amplified events. We conjecture that this apparent discrepancy can plausibly be explained by the relative quality of the data sets. In particular, relative to the MDM data set, the INT data cover a much ( $>3$ times) larger portion of the sky around the M 31 bulge, with more than the double the nights of observation. In particular, in the MDM Control field, with at best one point every 3 nights, we can obviously not characterize events of such short duration. On the other hand, we point out that while the POINTAGAPE analysis has been carried out with a threshold on the magnitude at maximum of the detected variations $\left(R_{\max }<21\right)$, in the analysis presented here we do not adopt such a threshold (we recall, see Paper I, that we are sensitive to variations down to around $R_{\max } \sim 23$ ).

\section{Discussion and perspectives}

We have reported on the status of the analysis of the MDM data carried out in the framework of a search for microlensing events towards M 31. As a result of a full analysis of 2 years of data, in which we have also taken advantage of the longer baseline made available by the use of some INT data, we have first excluded as viable all the microlensing candidates previously reported in Paper I. Second, we have reported the selection of three more candidates compatible with a Paczyński light curve, which may possibly be due to self lensing. On examining their extensions into the INT data, we find a second variation in both cases for which such data are available. However, these variations are strikingly different from and much longer than the short-duration events seen in the MDM data. Hence, it is plausible that each of these second variations is due to an unrelated variable star superimposed on a true microlensing signal. The available data do not permit us to resolve this question.
We have discussed the issue of variable-star contamination of the signal: we note that this is a significantly more serious problem than in the cases of the search for microlensing events towards the Magellanic clouds and the Galactic bulge in which one monitors the flux variations of resolved sources of known type.

The analysis reported in this paper shows once again the crucial role played by a frequent sampling of the data and the total baseline length, both of which are essential in getting meaningful results when extracting microlensing signals from the background of variable sources. In this light, the forthcoming full analysis of the detection efficiency, together with results from the new sets of data acquired in the 2001 and 2002 seasons at both $1.3 \mathrm{~m}$ and $2.4 \mathrm{~m}$ MDM telescopes with a new $8 \mathrm{~K}-\mathrm{CCD}$ array (as well as the prospective 2003 campaign at the TT1 telescope in the south of Italy, Bozza et al. 1999), should eventually give us the opportunity to draw firmer conclusions on the issue of the MACHO fraction in the halo of M 31 galaxy.

Acknowledgements. We thank the referee for useful comments and suggestions. We are grateful to the POINT-AGAPE collaboration for allowing us the access to their data set. SCN was supported by the Swiss National Science Foundation and by the Tomalla Foundation. AG was supported by grant 02-01266 from the US NSF.

\section{References}

Alcock, C., Allsman, R. A., Alves, D. R., et al. 2000, ApJ, 542, 281

Ansari, R., Aurière, M., Baillon, P., et al. 1997, A\&A, 324, 843

Ansari, R., Aurière, M., Baillon, P., et al. 1999, A\&A, 344, L49

Aurière, M., Baillon, P., Bouquet, A., et al. 2001, ApJ, 553, L137

Baillon, P., Bouquet, A., Giraud-Héraud, Y., \& Kaplan, J. 1993, A\&A, 277,1

Bozza, V., Calchi Novati, S., Capaccioli, M., et al. 1999 [astro-ph/9907162]

Calchi Novati, S., Iovane, G., Marino, A. A., et al. 2002, A\&A, 381, 848 (Paper I)

Crotts, A. P. 1992, ApJ, 399, L43

Crotts, A. P., Uglesich, R., Gould, A., et al. 2000 [astro-ph/0006282]

Durbin, J., \& Watson, G. S. 1951, Biometrika, 38, 159

Gould, A. 1996, ApJ, 470, 201

Jetzer, Ph. 1994, A\&A, 286, 426

Jetzer, Ph., Mancini, L., \& Scarpetta, G. 2002, A\&A, 393, 129

Magnier, E. A., Lewin, W. H. G., van Paradijs, J., et al. 1992, A\&AS, 96,379

Lasserre, T., Afonso, C., Albert, J. N., et al. 2000, A\&A, 355, L39

Paczyński, B. 1986, ApJ, 304, 1

Paulin-Henriksson, S., Baillon, P., Bouquet, A., et al. 2002, ApJ, 576, L121

Paulin-Henriksson, S., Baillon, P., Bouquet, A., et al. 2003, A\&A, 405, 15

Riffeser, A., Fliri, J., Gössl, C. A., et al. 2001, A\&A, 379, 362

Tomaney, A., \& Crotts, A. 1996, AJ, 112, 2872 